\title{
Wavelength dependence of polarisation of photoelectrons ejected by unpolarised vUV radiation from argon and krypton atoms
}

\author{
U Heinzmann, G Schönhense and J Kessler \\ Physikalisches Institut der Universität Münster, D-4400 Münster, West Germany
}

Received 18 December 1979

\begin{abstract}
The spin polarisation of the photoelectrons emitted by argon and krypton atoms exposed to unpolarised VUV radiation has been measured at rare-gas resonance wavelengths in the energy range from the threshold up to $41 \mathrm{eV}$. Comparison of the wavelength dependence of the measured polarisation with theoretical results shows good agreement.
\end{abstract}

One of the novelties in photoionisation is the fact that photoelectrons ejected from unpolarised atoms can be spin polarised even if the light is unpolarised. After this had been predicted theoretically for certain types of atoms by Lee (1974) and Cherepkov $(1974,1978)$, the experimental verification has been performed for lead atoms (Heinzmann et al 1978) and for xenon atoms (Heinzmann et al 1979). It is necessary in such experiments to detect only photoelectrons emerging in well defined directions since, unlike photoionisation with circularly polarised light (see for example Kessler 1976), the electron polarisation would vanish if all the photoelectrons were extracted independently of their direction of emission.

It is the purpose of this letter to report detailed experimental results for argon and krypton atoms and to compare the measured wavelength dependence of the polarisation with theoretical predictions by Cherepkov (1979) (random phase approximation with exchange) and by Huang et al (1979) (relativistic random phase approximation).

A schematic diagram of the apparatus used is shown in figure 1 . The radiation is produced by means of capillary discharge tubes (Heinzmann and Schönhense 1979, 1980), yielding very intense helium, neon and argon resonance lines (He r: $10^{13}$ photons/s at the target). The VUV light crosses the atomic beam in a region free of electric and magnetic fields. The photoelectrons produced pass through an electron spectrometer (cylindrical mirror analyser, energy resolution $0.7 \%$ ) and are focused onto the entrance of a $120 \mathrm{keV}$ accelerating tube. The spin polarisation which is perpendicular to the reaction plane of the photoionisation process is determined from the left-right asymmetry of the electron intensity scattered through $120^{\circ}$ by the gold foil of a Mott detector.

The angular dependence of the photoelectron polarisation has been predicted by Lee (1974) and by Cherepkov (1974) and been measured by Schönhense (1980) to be

$$
P(\theta)=\frac{2 \xi \hat{\boldsymbol{s}} \cdot\left(\hat{\boldsymbol{k}}_{i} \times \hat{\boldsymbol{k}}_{0}\right)\left(\hat{\boldsymbol{k}}_{i} \cdot \hat{\boldsymbol{k}}_{0}\right)}{1-\frac{1}{2} \beta\left[\frac{3}{2}\left(\hat{\boldsymbol{k}}_{i} \cdot \hat{\boldsymbol{k}}_{0}\right)^{2}-\frac{1}{2}\right]}=\frac{2 \xi \sin \theta \cos \theta}{1-\frac{1}{2} \beta P_{2}(\cos \theta)}
$$




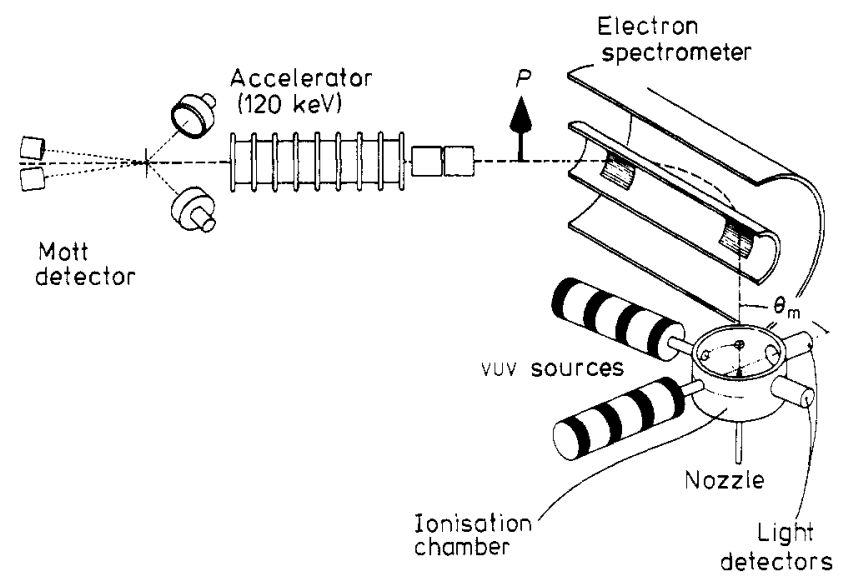

Figure 1. Schematic diagram of the apparatus.
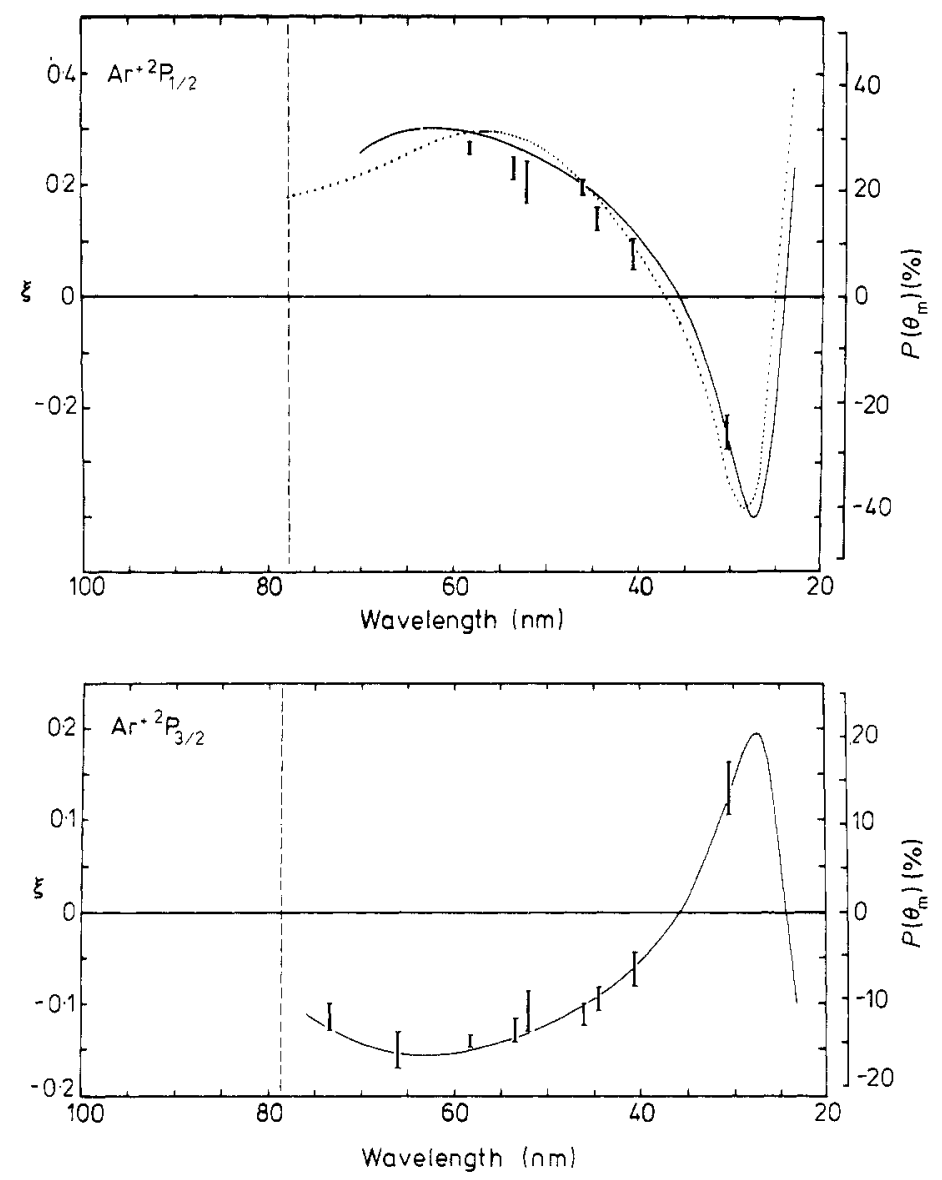

Figure 2. Photoelectron polarisation produced by unpolarised light from argon atoms: experimental values with error bars and theoretical data by Cherepkov (1979) (dotted curve) and Huang et al (1979) (full curves). The broken lines represent the photoionisation thresholds. 
where $\hat{\boldsymbol{s}}=\left(\hat{\boldsymbol{k}}_{i} \times \hat{\boldsymbol{k}}_{0}\right) / \sin \theta$ is a unit vector fixed in space and the polarisation vector is $\boldsymbol{P}(\theta)=\boldsymbol{P}(\theta) \hat{\boldsymbol{s}}, \hat{\boldsymbol{k}}_{i}$ and $\hat{\boldsymbol{k}}_{0}$ are the unit vectors of the directions of the incident radiation and the electron momentum, respectively, and $\theta$ is the angle between $\hat{\boldsymbol{k}}_{i}$ and $\hat{\boldsymbol{k}}_{0}$.

In the present experiment the angles between the radiation emitted by the two vUV sources and the photoelectrons observed are $\theta_{\mathrm{m}}=54^{\circ} 44^{\prime}$ and $\pi-\theta_{\mathrm{m}}$, respectively. Since at these angles the Legendre polynomial $P_{2}(\cos \theta)$ disappears, the asymmetry parameter $\beta$ in the denominator, which is proportional to the differential photoionisation cross section, does not play a role when $\xi$ is determined from the measured polarisation. The above formula shows that switching from one vUV source to the other reverses the photoelectron polarisation. This procedure was repeated once a minute and allowed elimination of instrumental asymmetries. The forward counters in the Mott detector allow additional corrections for these asymmetries to be made.

Figures 2 and 3 show the parameters $\xi$ and the measured polarisations as functions of the wavelength for $\mathrm{Ar}$ and $\mathrm{Kr}$. The polarisation of photoelectrons associated with the ${ }^{2} \mathrm{P}_{1 / 2}$ and ${ }^{2} \mathrm{P}_{3 / 2}$ states of the residual ions differs in sign for both atoms, which shows the necessity of resolving the fine structure by use of the electron spectrometer. Otherwise the polarisations would almost cancel one another. The error bars in figures 2 and 3 represent the single statistical error of the polarisation analysis including the uncertainty of the analysing power of the Mott detector (Sherman function).
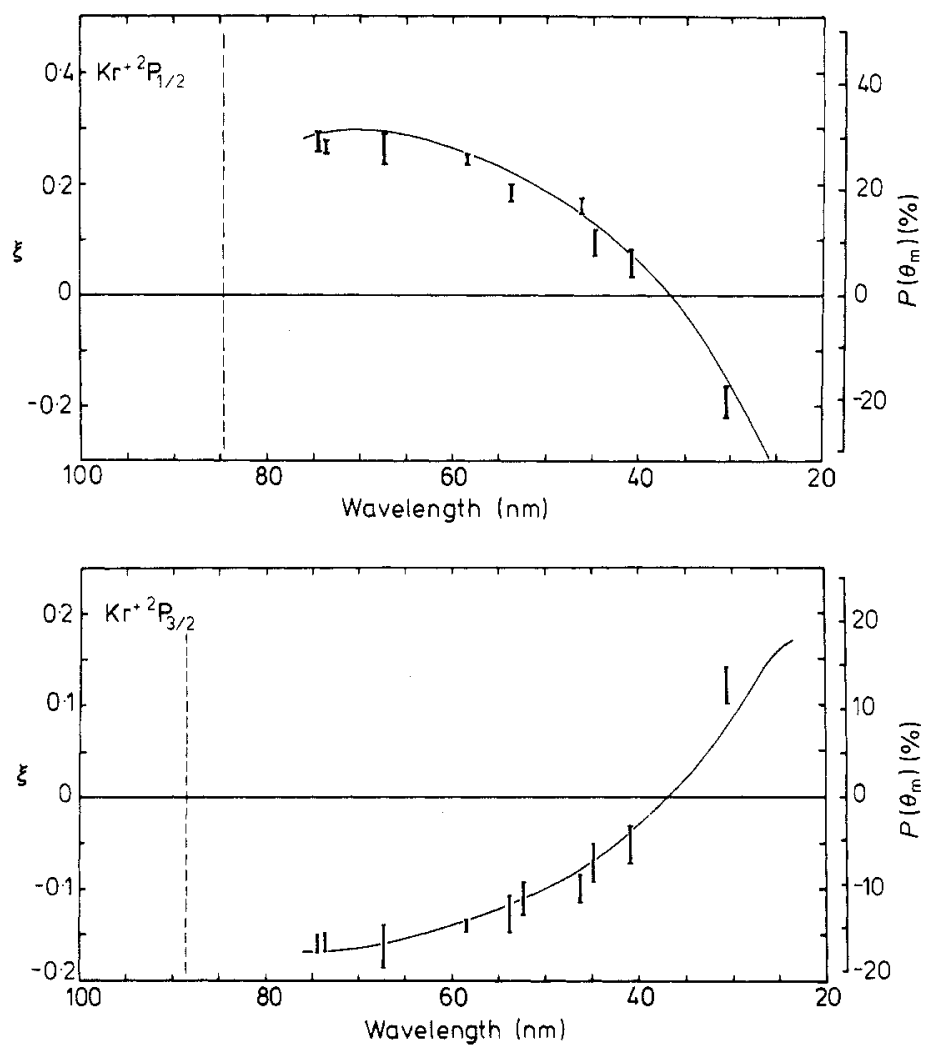

Figure 3. Photoelectron polarisation produced by unpolarised light from krypton atoms: experimental values with error bars and theoretical data by Huang et al (1979). The broken lines represent the photoionisation thresholds. 
Figures 2 and 3 also show a few theoretical results. While the dotted curve calculated by Cherepkov (1979) using a random phase approximation with exchange gives the wavelength dependence of $\xi$ only for the case of $\mathrm{Ar}^{+2} \mathrm{P}_{1 / 2}$, Huang et al (1979) have calculated $\xi$ for both ionic states of argon and krypton (full curves). It is worth noting that the curve of Cherepkov in figure 2 does not agree in sign with his published polarisation data because there was an error in sign (N A Cherepkov 1979, private communication). Qualitatively, in the case of $\mathrm{Ar}^{+2} \mathrm{P}_{1 / 2}$, both theoretical curves show within the error limits good agreement with the experimental results. Quantitatively, the results of the relativistic random phase approximation by Huang et al (1979) seem to agree better. This indicates that relativistic effects like the influence of spin-orbit interaction on the continuum wavefunctions should not be neglected even for an atom of small atomic number like argon. The agreement between the experimental and the theoretical wavelength dependence of $\xi$ for krypton atoms is very good as shown in figure 3 .

Support by Deutsche Forschungsgemeinschaft is gratefully acknowledged.

\section{References}

Cherepkov N A 1974 Sov. Phys.-JETP 38 463-9

- 1978 J. Phys. B: Atom. Molec. Phys. 11 L435-9

1979 J. Phys. B: Atom. Molec. Phys. 12 1279-96

Heinzmann U and Schönhense G 1979 Proc. 11th Int. Conf. on the Physics of Electronic and Atomic Collisions (Kyoto: Society for Atomic Collision Research) Abstracts p 24

1980 to be published

Heinzmann U, Schönhense G and Kessler J 1979 Phys. Rev. Lett. 42 1603-5

Heinzmann U, Schönhense G and Wolcke A 1978 Proc. Int. Workshop on Coherence and Correlation in Atomic Collisions ed H Kleinpoppen and J F Williams (New York: Plenum) pp 607-12 in press

Huang K-N, Johnson W R and Cheng K T 1979 Phys. Rev. Lett. 43 1658-61

Kessler J 1976 Polarized Electrons (Berlin: Springer) pp 123-46

Lee C M 1974 Phys. Rev. A 10 1598-604

Schönhense G 1980 to be published 